\title{
RINGS HAVING SOLVABLE ADJOINT GROUPS
}

\author{
P. B. BHATTACHARYA AND S. K. JAIN
}

\begin{abstract}
Let ${ }^{\circ} R$ denote the group of quasi-regular elements of a ring $R$ with respect to circle operation. The following results have been proved: (1) If $R$ is a perfect ring and ${ }^{\circ} R$ is finitely generated solvable group then $R$ is finite and hence ${ }^{\circ} R=P_{1} \circ P_{2} \circ \cdots \circ P_{m}$ where $P_{i}$ are pairwise commuting p-groups. (2) Let $R$ be a locally matrix ring or a prime ring with nonzero socle. Then ${ }^{\circ} R$ is solvable iff $R$ is either a field or a $2 \times 2$ matrix ring over a field having at most 3 elements.
\end{abstract}

For a ring $R$ let $J(R)$ denote the Jacobson radical, ${ }^{\circ} R$ the group of quasi-regular elements with respect to circle operation and $U(R)$ the group of units if $R$ has identity. We know that if $R$ has identity, then ${ }^{\circ} R$ is isomorphic to $U(R) .{ }^{\circ} R$ is called the adjoint group of $R$. The object of this paper is to study certain classes of rings $R$ for which ${ }^{\circ} R$ is nilpotent, supersolvable or solvable.

1.1. Let $M$ be a unital free $R$-module over a ring $R$ in which 2 is invertible and let $U(S)$ be supersolvable where $S=\operatorname{Hom}_{R}(M, M)$. Then $S=R$.

If $S \neq R$, then $S$ contains a copy $T$ of a $2 \times 2$ matrix ring over $R$. In this case we choose $a, b, c \in U(T)$ such that

$$
a=\left(\begin{array}{ll}
1 & 1 \\
0 & 1
\end{array}\right), \quad b=\left(\begin{array}{ll}
2 & 1 \\
0 & 4
\end{array}\right), \quad c=\left(\begin{array}{ll}
-1 & \frac{1}{2} \\
-2 & 1
\end{array}\right) .
$$

Direct computation yields $a^{-1} b^{-1} a b=a, b^{-1} c^{-1} b c=b$. These relations first imply that $a, b$ belong to the derived group of $U(T)$ and further the relation $a^{-1} b^{-1} a b=a$ implies that the derived group cannot be nilpotent. Hence $U(T)$ cannot be supersolvable in contradiction to the hypothesis that $U(S)$ is supersolvable. This proves 1.1 .

The following example shows that if $2=0$ in a ring $R$ then 1.1 may not be true: The group of units of a $2 \times 2$ matrix ring over a field of 2 elements is supersolvable.

1.2. Let $M$ be a unital free module over a ring $R$ of characteristic 2 and let the group $U(S)$ be nilpotent where $S=\operatorname{Hom}_{R}(M, M)$. Then $S=R$.

Received by the editors February 6, 1969 and, in revised form, November 13, 1969. AMS 1970 Subject Classifications. Primary 16A26.

Key Words and Phrases. Perfect rings, locally matrix rings, prime rings with nonzero socles, solvable adjoint groups. 
We argue as in 1.1. Here we choose

$$
a=\left(\begin{array}{ll}
0 & 1 \\
1 & 1
\end{array}\right), \quad b=\left(\begin{array}{ll}
1 & 0 \\
1 & 1
\end{array}\right) .
$$

Again $a^{-1} b^{-1} a b=a$ and this shows that the subgroup consisting of all the $2 \times 2$ matrices over $R$ cannot be nilpotent, in contradiction to the hypothesis that the group $U(S)$ is nilpotent. Hence $S=R$.

1.3. (Hua). If $D$ is a division ring and ${ }^{\circ} D$ is solvable then $D$ is a field.

1.4. The group of units of the ring of all linear transformations of a vector space $V$ over a division ring $D$ is nilpotent iff $\operatorname{dim}_{D} V=1$ and $D$ is a field.

Since nilpotent groups are solvable the result follows from 1.2 and 1.3 .

1.5. (Scott). Let $F$ be a field, $G=\operatorname{SL}(2, F)$ and $\#(F)>3$. Then $G=G^{1}$.

1.6. (Dickson). The group of quasi-regular elements in the $3 \times 3$ matrix ring over a field having at most 3 elements is not solvable.

1.7. The group of units of the ring of all linear transformations of a vector space $V$ over a division ring $D$ is solvable iff either $D$ is any field and $\operatorname{dim}_{D} V=1$ or $D$ is a field having at most 3 elements and $\operatorname{dim}_{D} V=2$.

This is a consequence of $1.3,1.5$ and 1.6.

1.8. If $R$ is any ring such that $R \mid J(R)$ is artinian and ${ }^{\circ} R$ is solvable then $R \mid J(R)$ is a finite direct sum of rings $R_{i}$ where $R_{i}$ is a field or a $2 \times 2$ matrix ring over a field having at most 3 elements.

Since homomorphic image of a solvable group is solvable, the result follows from 1.7 .

1.9. (Bass). $R$ is a ring with dcc for principal right ideals iff $R \mid J(R)$ is artinian and $J(R)$ is $T$-nilpotent.

Such rings have been called perfect rings by Bass.

1.10. If $R$ is a perfect ring and ${ }^{\circ} R$ is a finitely generated solvable group then $R$ is finite, and hence ${ }^{\circ} R=P_{1} \circ \cdots \circ P_{m}$ where $P_{i}$ are pairwise commuting $p$-groups.

Since the fields whose multiplicative groups are finitely generated are Galois fields, finiteness of $R \mid J(R)$ follows at once from 1.8 and 1.9. This implies ${ }^{\circ} J(R)$ is also finitely generated. Further $J(R)$ is locally nilpotent since it is $T$-nilpotent. Hence $J(R)$ is nilpotent which implies ${ }^{\circ} J(R)$ is nilpotent and therefore by Watters $J(R)^{+}$is finitely generated. Since $R$ is perfect, we get $J(R)$ is finite. Hence $R$ is finite. The last assertion is a consequence of Hall's well-known theorem for finite solvable groups. 
2. We now proceed to characterise the class of prime rings, with nonzero socles, for which ${ }^{\circ} R$ is solvable.

We denote by $C$ the class of all rings which are either fields or $2 \times 2$ matrix rings over fields having at most 3 elements. In what follows it is assumed that ${ }^{\circ} R$ is solvable.

2.1. A locally matrix ring $R$ over a division ring is in $C$.

We recall that $R$ is a locally matrix ring over a division ring $\Delta$ if each finite subset is in a subring of $R$ isomorphic to an $n \times n$ matrix ring over $\Delta$, for some natural number $n$. If $R$ is not a $2 \times 2$ matrix ring over a field having at most 3 elements or it is not a field having less than 82 elements then $R$ must contain at least 82 distinct elements $a_{i}$. These $a_{i}$ can be imbedded in a subring $S$ of $R$ where $S$ is an $n \times n$ matrix ring over $\Delta$. Since ${ }^{\circ} S$ is a subgroup of ${ }^{\circ} R$, the subgroup ${ }^{\circ} S$ is also solvable. By 1.7 we get that $S \cong \Delta$ is a commutative field. Since $S$ contains arbitrary elements of $R$, we get $R$ is commutative and hence $R$ is a field proving that $R$ is in $C$.

Since by Litoff theorem simple ring with minimal one-sided ideals is a locally matrix ring over a division ring 2.1 gives

Corollary. Simple rings with minimal one-sided ideals are in the class $C$.

2.2. If $R$ is a prime ring with nonzero socle then $R$ is in $C$. We know that $R$ is then a primitive ring with nonzero socle.

The socle is a simple ring with minimal one-sided ideals. Thus by 2.1 socle (as a ring) has identity. But then the identity of the socle (which is also an ideal in $R$ ) is easily shown to be the identity for the whole ring. Hence $R=$ socle, proving that $R$ is in the class $C$.

\section{REFERENCES}

1. Hyman Bass, Finitistic dimension and a homological generalization of semiprimary rings, Trans. Amer. Math. Soc. 95 (1960), 466-488. MR 28 \#1212.

2. L. Hua, On the multiplicative group of a field, Acad. Sinica Science Record 3 (1950), 1-6. MR 12, 584.

3. Nathan Jacobson, Structure of rings, 2nd rev. ed., Amer. Math. Soc. Colloq. Publ., vol. 37, Amer. Math. Soc., Providence, R. I., 1964. MR 36 \#5158.

4. W. R. Scott, Group theory, Prentice-Hall, Englewood Cliffs, N. J., 1964. MR 29 $\# 4785$.

5. J. F. Watters, On the adjoint group of a radical ring, J. London Math. Soc. 43 (1968), 725-729. MR 37 \#5251.

Ohio University, Athens, Ohio 45810 